\title{
A case of tetanus secondary to an odontogenic infection
}

\author{
Majid Darraj, MD*; James Stone, $\mathrm{BSc}^{\dagger}$; Yoav Keynan, MD, $\mathrm{PhD}^{\ddagger *}$; Kristjan Thompson, $\mathrm{MD}^{\dagger}$; \\ Carolyn Snider, MD, MPH ${ }^{\delta \mathbb{T}_{* *}}$
}

\begin{abstract}
Tetanus is a life-threatening clinical syndrome that commonly presents with muscular spasms, rigidity, and autonomic instability. It is considered rare in industrialized countries, and tetanus occurring secondary to dental abscesses, procedures, or infections has been infrequently reported. We describe the case of a patient inadequately immunized for tetanus, who presented to the emergency department with muscular spasms, rigidity, and autonomic instability in the setting of an odontogenic infection. A clinical diagnosis of tetanus was made and subsequently managed successfully.
\end{abstract}

\section{RÉSUMÉ}

Le tétanos est une maladie clinique, potentiellement mortelle, qui s'accompagne souvent de spasmes musculaires, de rigidité et d'une instabilité du système nerveux autonome. Il s'agit d'une maladie rare dans les pays industrialisés, et les cas de tétanos consécutifs à des abcès dentaires, à une intervention ou à des infections ne font pas souvent l'objet de description dans la documentation médicale. Sera exposé ici un cas de tétanos chez un malade non suffisamment immunisé contre la maladie, qui a consulté un médecin au service des urgences pour des spasmes musculaires, de la rigidité et une instabilité $\mathrm{du}$ système nerveux autonome, dans le contexte d'une infection odontogène. Un diagnostic clinique de tétanos a été posé et la maladie a été traitée adéquatement.

Keywords: tetanus, dental, immunization

\section{CASE REPORT}

A healthy 32-year-old male presented to the emergency department with generalized body muscular spasms and a 2-week history of right upper-third molar tooth pain due to a recent tooth fracture in the setting of multiple dental caries and baseline poor dentition.

He reported 48 hours of intermittent episodes of jaw twitching lasting for approximately 10 minutes, re-occurring every 15 minutes. Thereafter, the spasms spread to involve the neck, shoulder, arms, back, and legs with increasing intensity and duration. He described the spasms as extremely painful.

The patient had no other significant past medical history and denied illicit drug use. The patient was a roof mechanic. He denied any recent lacerations. The immunization records revealed that he was inadequately immunized and was last vaccinated against tetanus 12 years previous.

Examination revealed moderate swelling and erythema surrounding the right upper-third molar site that was fractured. The spasms involved the majority of facial muscles causing trismus (lockjaw due to spasm of the muscles of mastication), dysphagia, and eyelid closure. Spasms of the neck and back resulted in stiffness, involuntary extension, and arching; and of the upper and lower limbs causing various extension and flexion movements. There was no impairment of consciousness during the patient's entire presentation, and in between episodes he had normal power and sensation throughout; however, he had increased muscular tone and widespread exaggerated reflexes with elicited Babinski reflexes bilaterally. Despite the patient's increased nuchal muscle tone, he did not exhibit other signs of meningitis. He was intermittently diaphoretic yet afebrile, with blood pressures ranging between 100 and $180 \mathrm{~mm} \mathrm{Hg}$ systolic, heart rate between 78 and 90 beats per minute, and normal oxygenation. He never exhibited any signs of hemodynamic instability or respiratory distress. Cardiopulmonary and abdominal examinations were within normal limits.

From the *Department of Medical Microbiology, †Department of Health Sciences, $\ddagger$ Department of Internal Medicine, §Department of Emergency Medicine, and IDepartment of Community Health Sciences, College of Medicine, University of Manitoba, Winnipeg, MB; and ${ }^{* *}$ Children's Hospital Research Institute of Manitoba, Winnipeg, MB.

Correspondence to: Dr. Carolyn E. Snider, University of Manitoba,656A-715 McDermot Ave., Winnipeg, MB R3M 1E1; Email: csnider@chrim.ca 
A video was recorded of this patient with spastic upper-body contractions and exhibiting symptoms consistent with lockjaw (see Supplemental Material).

Initial investigations were non-contributory, including complete blood count, extensive metabolic panel, and drug and toxin screening. Computed tomography scans of the neck, brain, and sinuses did not show any evidence of abscesses or osteomyelitis.

In addition to clinical tetanus, our differential diagnosis for the patient's upper-body spasms and trismus included dystonic reaction, toxicological sequelae (neuroleptic malignant syndrome, serotonin syndrome, strychnine, etc.), deep space dental infection, or meningitis. Based on the patient's history, incomplete immunization status, and, in the setting of an odontogenic infection with a triad of rigidity, painless muscular spasms and signs of autonomic instability, a clinical diagnosis of tetanus was made. The patient was promptly treated with intramuscular human anti-tetanus immunoglobulin (TIG), intravenous (IV) ceftriaxone and metronidazole, and IV diazepam as needed. Ketorolac was given for analgesia, and the patient was admitted to the intensive care unit for monitoring. Subsequently, oral-maxillary surgery was consulted, and the infected tooth was successfully extracted for source control. Wound swabs from the site of the extracted tooth were sent for culture and sensitivity, however, failed to grow any organism. Over the following 7 days, the patient's spasms significantly decreased, and, upon discharge, IV ceftriaxone and metronidazole were substituted with oral amoxicillin-clavulanic acid to complete a total course of 10 days of antibiotics.

\section{DISCUSSION}

Tetanus is a life-threatening neuromuscular disorder characterized by acute muscular spasms that are caused by the toxin-producing Clostridium tetani (C. tetani). ${ }^{1}$ Although still a common source of infection in the developing world, tetanus has become rare in industrialized and Western nations as a result of effective childhood vaccinations. Tetanus commonly presents with trismus and dysphagia, later evolving to include muscular spasms, rigidity, and autonomic instability as the disease progresses. ${ }^{1,2}$ Tetanus typically occurs after a small laceration or puncture wound in patients has been exposed to contaminated soil or metallic objects, which, although rare, can be traced back to transmission from surgical procedures, insect bites, IV drug use, and dental infections. ${ }^{3-5}$

C. tetani is an anaerobic, gram-positive bacillus. When it matures, it forms spores that can enter the body through an area of skin breakdown or a contaminated wound. The spores can be found within soil and in animal feces worldwide. Bacilli are formed from the spores after a short period of incubation and subsequently release two exotoxins: tetanospasmin and tetanolysin. Tetanospasmin, the toxin responsible for the clinical effects of $C$. tetani, enters the presynaptic terminal of neuromuscular end plates, exerting its neurotoxic effects resulting in prolonged contraction of muscle fibres. It acts on the inhibitory GABA-ergic neurons by blocking these neuronal receptors, and prevents the inhibition of motor reflexes, thereby resulting in spastic and rigid contractions. ${ }^{1,5}$

The clinical definition of tetanus according to the Centers for Disease Control and Prevention and the World Health Organization includes an acute illness with muscular spasms and rigidity in the absence of alternative diagnosis. ${ }^{1,6}$ Tetanus is strictly a clinical diagnosis because $C$. tetani is recovered from wounds in only $30 \%$ of cases and also can be recovered from patients without tetanus. ${ }^{6}$ Therefore, and as in the case of our patient, a negative culture for $C$. tetani should not be used to rule out the diagnosis of tetanus, especially when the patient's clinical history, signs, and symptoms are suggestive of the disease.

Tetanus vaccination is recommended for adults every 10 years; however, it is important to realize that tetanus can occur despite previous immunization and protective anti-tetanus antibody levels. ${ }^{6}$ Although cultures collected from our patient failed to isolate C. tetani, we feel that the patient's clinical presentation fit the case definition of clinical tetanus. Some clinicians who are not familiar with tetanus may have the misconception that immunized individuals do not develop clinical tetanus, which could delay diagnosis and treatment, resulting in a potential increase in both morbidity and mortality.

Tetanus occurring secondary to an odontogenic source has been documented in a few case reports only. The first case report describing tetanus following tooth extractions was reported in 1949 and was thought to be from contaminated instrumentation. Since then, there have been limited cases of tetanus following dental procedures with the implementation of strict sterilization practices. Reports over the years linking cases of tetanus to oral or dental origins include tooth 
extractions, root canals, caries, abscesses, and oral trauma; however, in up to $40 \%$ of cases, a definitive source of infection cannot be identified. ${ }^{5,7}$ Typically, these cases involve unimmunized individuals or those in developing countries. ${ }^{8}$ However, disease in an immunocompetent individual who was adequately immunized has been previously reported. ${ }^{4,9}$

There are four crucial principles in the management of tetanus, including sedation and potentially even paralysis to control autonomic dysfunction and violent muscular spasms with propofol, neuromuscular blocking agents, or benzodiazepines; neutralization of the neurotoxin by human anti-TIG; antibiotics and surgical debridement of the potential source (if known); and stabilization/monitoring in the intensive care unit. Either penicillin or metronidazole can be used as first-line antibiotics for the treatment of a tetanus infection., We elected to give broad antibiotic coverage because odontogenic infections are often polymicrobial.

Tetanus cases in industrialized countries are rare because of comprehensive immunization programs; however, the mortality rates among those select few cases remain quite high. In Canada, 42 cases of tetanus were reported between 2000 and 2013 with an average of 3 cases per year, resulting in a total of 6 deaths. The immunization status of these patients is not known, and there were no reported neonatal cases.

\section{CONCLUSION}

Because of overwhelmingly successful and widespread immunization programs, tetanus is rare in many industrialized countries. However, sporadic cases still occur with high mortality rates. Therefore, it is crucial for clinicians to identify and promptly treat the disease in patients presenting with clinical signs, symptoms, and histories consistent with tetanus, particularly in patients with inadequate immunization.
We attribute the response to therapy and prompt an uneventful recovery of our patient to the early diagnosis, prompt treatment, definitive source control, and the speculated presence of partial immunity offered by remote vaccination.

\section{SUPPLEMENTARY MATERIAL}

To view supplementary material for this article, please visit https://doi.org/10.1017/cem.2016.390

Competing interests: None declared.

\section{REFERENCES}

1. Afshar M, Raju M, Ansell D, et al. Narrative review: tetanus a health threat after natural disasters in developing countries. Ann Intern Med 2011;154:329.

2. Rodrigo C, Fernando D, Rajapakse S. Pharmacological management of tetanus: an evidence-based review. Crit Care 2014; 18(2):217.

3. Paterson AW, Ryan W, Rao-Mudigonda VV. Trismus: or is it tetanus? A report of a case. Oral Surg Oral Med Oral Pathol Oral Radiol Endodontol 2006;101(4):437-41.

4. Felter RA, Zinns LE. Cephalic tetanus in an immunized teenager: an unusual case report. Pediatr Emerg Care 2015; 31(7):511-3.

5. Smith AT, Drew SJ. Tetanus: a case report and review. 7 Oral Maxillofac Surg 1989;53:77-80.

6. Centers for Disease Control and Prevention. Vaccine and immunizations. Chapter 16: tetanus. Available at: http://www. cdc.gov/vaccines/pubs/surv-manual/chpt16-tetanus.html (accessed 14 February 2016).

7. Cone LA, Fensterer FS. Case reports. Tetanus following a dental operation. Calif Med 1973;119(3):57-8.

8. Ajayi E, Obimakinde O. Cephalic tetanus following tooth extraction in a Nigerian woman. 7 Neurosci Rural Pract 2011;2(2):201-2.

9. Konig K, Ringe H, Dorner BG. Atypical tetanus in a completely immunized 14-year-old boy. Pediatrics 2007;120: e1355-8. 